\title{
Miscibility of Chitosans/Polyamide 6 Blends
}

\author{
Min Jae Ko, Won Ho Jo, ${ }^{\dagger}$ Ho Cheol Kim, ${ }^{*}$ and Sang Cheol LeE** \\ Department of Fiber and Polymer Science, Seoul National University, \\ Seoul 151-742, Korea \\ * Central Research Institute, Hyosung T\&C, Anyang 430-080, Korea \\ ** Department of Polymer Science and Engineering, Kumoh National University of \\ Technology, Kumi 730-701, Korea
}

(Received April 23, 1997)

\begin{abstract}
Chitosans with different degrees of deacetylation were prepared by a chemical method, and miscibility between chitosan and polyamide 6 was examined as a function of the degree of deacetylation in chitosan. From dynamic mechanical analysis and solution viscosity measurement, it may be concluded that there are specific interactions between amorphous regions of both polymers and that the miscibility of the blends becomes better as the degree of deacetylation in chitosan decreases.
\end{abstract}

KEY WORDS Chitosan / Degree of Deacetylation / Polyamide 6/Blends / Miscibility /

Recently, more focus has been concentrated on natural polymers, because natural polymers are inexhaustible resources with biocompatible and biodegradable properties, and they do not cause environmental problems. Therefore, many blending systems containing natural polymers are being studied. ${ }^{1-15}$

Chitosan is an $N$-deacetylated product of chitin that is the second most abundant polysaccharide in nature. Moreover, it has very useful functional groups such as hydroxyl, amine and acetamide groups, and also shows antimicrobial activity. Thus, it is used in various fields ${ }^{11,12}$ ranging from a simple flocculant for waste water treatments to medical uses. In particular, interesting observations on the blends of chitosan with some synthetic polymers such as poly(vinyl alcohol), ${ }^{13}$ poly(vinylpyrrolidone), ${ }^{14}$ and poly(ethylene oxide) ${ }^{15}$ have been reported. Recently, Ratto et al. ${ }^{7}$ examined the miscibility of chitosan/polyamide blends and reported that the blends are partially miscible. But, they used solely a chitosan with $80 \%$ degree of deacetylation.

In this study, we control the degree of deacetylation of chitosan by a chemical method and investigate the miscibility of chitosan/polyamide 6 (PA6) blends as a function of the degree of deacetylation.

\section{EXPERIMENTAL}

\section{Materials}

Chitosan was purchased from Sigma Chemical Co., U.S.A. and purified by reprecipitation. ${ }^{16}$ Chitosan was dissolved in $5 \%$ acetic acid and filtered with stainless steel mesh. The solution was poured into sodium hydroxide solution, and the precipitates were washed and dried in a vacuum at $60^{\circ} \mathrm{C}$. The viscosity average molecular weight ${ }^{17}$ was determined by the Mark-Houwink equation: $[\eta]=K \bar{M}_{v}^{a}$ where $K=1.8 \times 10^{-3} \mathrm{~cm}^{3} \mathrm{~g}^{-1}$ in $0.1 \mathrm{M}$ acetic acid/0.2 $\mathrm{M} \mathrm{NaCl}$ solvent at $25^{\circ} \mathrm{C}$ and $a$ is 0.93 . The viscosity average molecular weight of the raw chitosan was $8.1 \times 10^{5} \mathrm{~g} \mathrm{~mol}^{-1}$. PA6 was supplied by Hyosung $\mathrm{T} \& \mathrm{C}$, Korea and used without purification.

† To whom correspondence should be addressed.
Blending samples were prepared by dissolving chitosan and PA6 in formic acid. The solution was cast on a polystyrene plate and the solvent was evaporated under reduced pressure.

\section{Deacetylation and Acetylation}

To prepare almost fully deacetylated chitosan, ${ }^{18}$ the purified chitosan with $80 \%$ deacetylation was deacetylated as following procedure: The chitosan was first treated with $47 \%$ sodium hydroxide solution at $110^{\circ} \mathrm{C}$ for $2 \mathrm{~h}$ under nitrogen atmosphere, and the sample was filtered and washed with hot water. This procedure was repeated twice and the sample was washed successively with water, methanol and ether. The degree of deacetylation $(D d)$ of the deacetylated sample was $95 \%$. Partially $N$-acetylated chitosans were prepared by the conventional method. ${ }^{19}$ The chitosan $(1 \mathrm{~g})$ with $95 \%$ Dd was dissolved in acetic acid $(100 \mathrm{~mL})$, and methanol $(100 \mathrm{~mL})$ was added to the solution. The mixed solution of acetic anhydride/methanol $(2 \% \mathrm{wt} / \mathrm{wt})$ was prepared separately, and was added to the chitosan solution drop by drop under vigorous stirring. The degree of deacetylation of chitosan was controlled by adding desired amount (e.g., $19.2 \mathrm{~mL}$ for $D d=68 \% ; 38.4 \mathrm{~mL}$ for $D d=57 \%$ ) of the mixed solution. After $1 \mathrm{~h}$ stirring, the solution was precipitated into excess methanol-ammonia $(7: 3, \mathrm{v} / \mathrm{v})$ solution. The precipitate was filtered off, washed successively with methanol and ether, and then dried at $60^{\circ} \mathrm{C}$ under vacuum.

\section{Characterizaztion of Chitosans and Their Blends}

The degree of deacetylation was determined by the titration method. ${ }^{20}$ Chitosan was dissolved in $0.5 \mathrm{~mol} \mathrm{~L}^{-1}$ of $0.3 \mathrm{~N} \mathrm{HCl}$. The solution was titrated with a standard solution of $0.1 \mathrm{~N} \mathrm{NaOH}$. There are two inflection points on the titration curve when $\mathrm{pH}$ is plotted against the amount of titrant $(0.1 \mathrm{~N} \mathrm{NaOH})$. The degree of deacetylation was calculated from the amount of $\mathrm{NaOH}$ consumed between two inflection points. The molecular weight and $D d$ of chitosans are listed in Table I. The number behind the sample code CD indicates the degree of deacetylation. 
Table I. Polymer characteristics

\begin{tabular}{cccc}
\hline Polymer & $\begin{array}{c}\text { Sample } \\
\text { code }\end{array}$ & $\begin{array}{c}\text { Dd } \\
\%\end{array}$ & $\begin{array}{c}\bar{M}_{v} \\
\mathrm{~g} \mathrm{~mol}^{-1}\end{array}$ \\
\hline Chitosan & CD95 & 95 & $7.0 \times 10^{5}$ \\
& CD80 & 80 & $8.1 \times 10^{5}$ \\
& CD68 & 68 & $5.5 \times 10^{5}$ \\
Polyamide 6 & CD57 & 57 & $7.0 \times 10^{5}$ \\
& PA6 & - & $6.0 \times 10^{4 \mathrm{a}}$
\end{tabular}

${ }^{\mathrm{a}} \bar{M}_{w}$ determined by GPC.

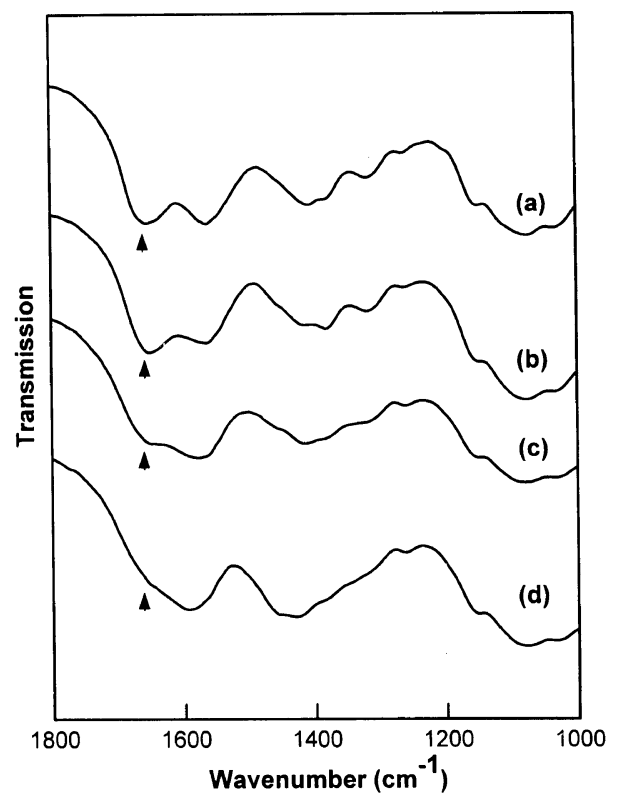

Figure 1. IR spectra of chitosans: (a) CD57; (b) CD68; (c) CD80; (d) CD95.

Figure 1 shows infrared spectra of chitosans obtained from a FT-IR spectrometer (Midac Co., U.S.A.). Amide I band at $1655 \mathrm{~cm}^{-1}$ disappeared characteristically with increasing the $D d$ owing to the transformation of acetamide groups into primary amine groups.

Change in crystalline state was monitored by a wide angle X-ray diffractometer (MAC MXP18A-HF), and thermal properties of chitosans and their blends were examined by a differential scanning calorimeter (Perkin Elmer, DSC-7). The samples were heated from room temperature to $250^{\circ} \mathrm{C}$ at a heating rate of $20^{\circ} \mathrm{C} \mathrm{min}^{-1}$. After quenching to room temperature, the samples were then reheated to $250^{\circ} \mathrm{C}$ at a heating rate of $20^{\circ} \mathrm{C} \mathrm{min}^{-1}$. The dynamic mechanical properties were measured with a dynamic mechanical thermal analyzer (Rheometric Scientific DMTA MK III) at $1 \mathrm{~Hz}$ over the temperature range from $-140^{\circ} \mathrm{C}$ to $220^{\circ} \mathrm{C}$ at a scan rate of $5^{\circ} \mathrm{C} \mathrm{min}{ }^{-1}$. Dynamic complex viscosity $\left(\eta^{*}\right)$ of blend solutions was measured on a Rheometric Mechanical Spectrometer (RMS 800) with a fixture of coni-cylinder geometry.

Antimicrobial activity of blending films was tested according to the shake flask method. ${ }^{21} \mathrm{~A}$ gram-positive bacteria, Staphylococcus aureus, was used for this testing. First, the number of living microorganisms was counted after stirring the microorganism suspension in an flask. The test solution was shaken for $1 \mathrm{~h}$ at $25^{\circ} \mathrm{C}$ after a blend film was added, and the number of living microorganisms was counted.

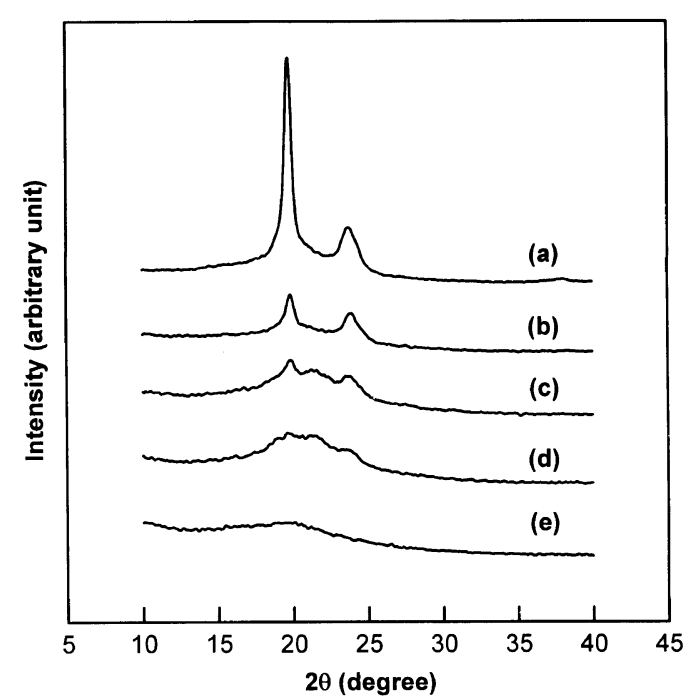

Figure 2. X-Ray diffraction patterns of CD57/PA6: (a) $0 / 100$; (b) 30/70; (c) $50 / 50$; (d) 70/30; (e) $100 / 0$.

\section{RESULTS AND DISCUSSION}

The crystal structure and crystallinity of chitosans/ PA6 blends were investigated as functions of the blend ratio. As shown in Figure 2, the chitosan (CD57) shows almost complete amorphous structure in agreement with the Samuels' results, ${ }^{22}$ whereas PA6 shows two strong diffraction peaks at $2 \theta=20^{\circ}$ and $24^{\circ}$. The former corresponds to (200) reflection and the latter to (002) and (202) reflections. This reflection indicates the $\alpha$-form of PA6. ${ }^{4,23}$ The two peaks corresponding to $\alpha$-form crystal gradually diffused with increasing chitosan content, but peak positions did not significantly change. However, at close examination an additional peak at $2 \theta=21.5^{\circ}$ is also observed. This peak results from (200), (001), and ( $\overline{2} 01)$ reflections of $\gamma$-form crystals of PA6. The $\gamma$-form crystals are usually observed in the X-ray diffraction pattern of PA6 films made from formic acid solution. ${ }^{24}$ The same was also observed for other chitosan/PA6 blends.

DSC thermograms of CD57/PA6 blends are shown in Figure 3. The blend samples show double melting peaks with different heights, resulting from PA6 crystals. This is well understood by considering the sequential process: partial melting, recrystallization and successive melting of recrystallized crystals in PA6. ${ }^{25,26}$ Significant change in melting temperature with the blend ratio was not observed unlike the melting behavior of miscible polymer blends. This may be partly due to the scale of homogeneity possibly being relatively large. When the heat of fusion of CD57/PA6 blends was plotted against the blend ratio, a linear relation was observed as shown in Figure 4, indicating that chitosan molecules do not suppress the growth of PA6 crystals in the blend. This is equivalent to the cellulose/PA6 blend. ${ }^{4}$ The glass transition temperature of both polymers was not detected as clearly as expected. From X-ray and DSC analysis, it is thought that chitosan does not significantly affect the crystallization of PA6.

As mentioned above, it is not adequate to determine the glass transition temperature of the blends by DSC. But it is well known that dynamic mechanical thermal 


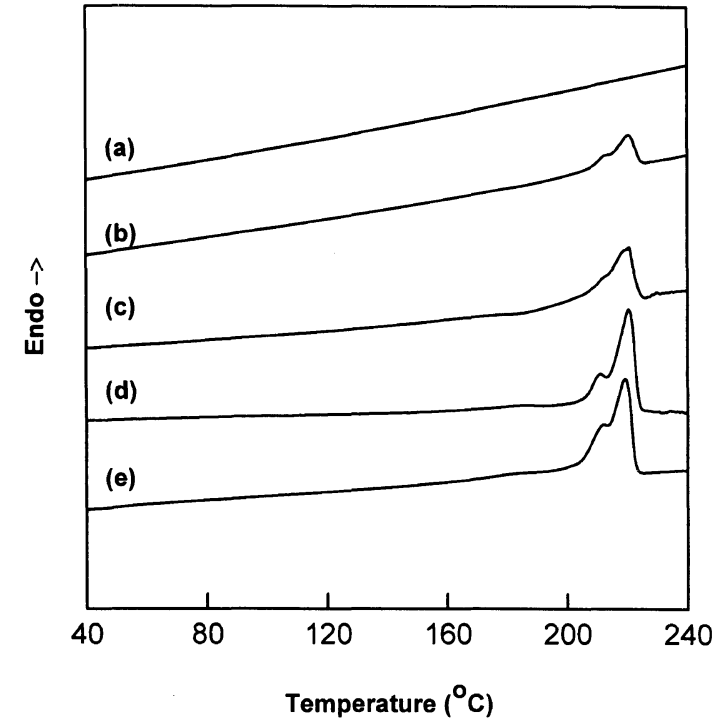

Figure 3. DSC thermograms of CD57/PA6 blends in the second scan: (a) $100 / 0$; (b) $70 / 30$; (c) $50 / 50$; (d) $30 / 70$; (e) $1 / 100$.
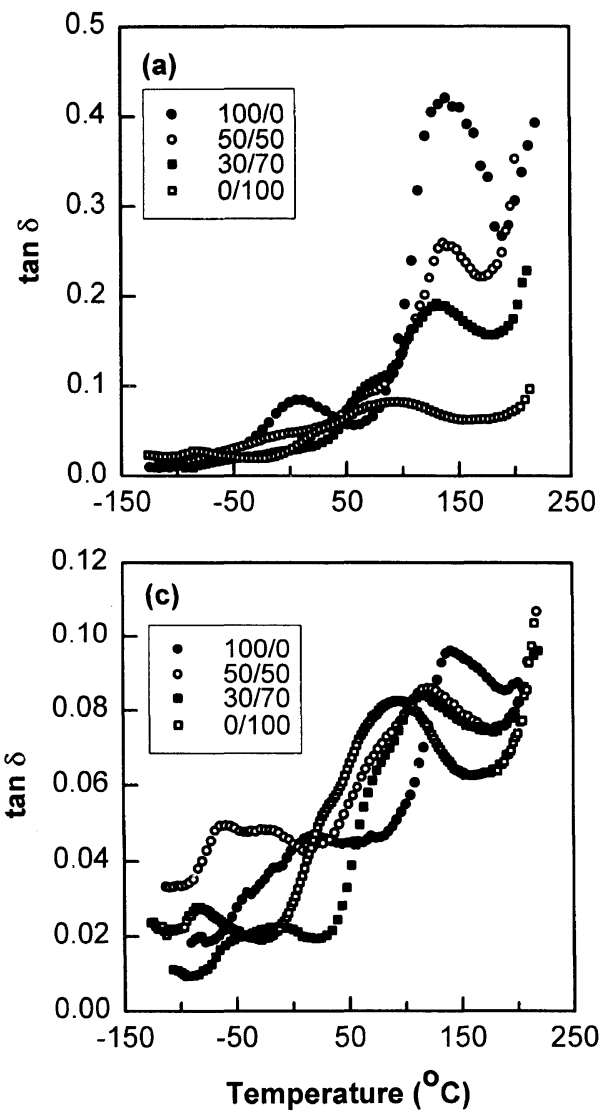

Figure 5. Temperature dependence of $\tan \delta$ for chitosan/PA6 blends: (a) CD95/PA6; (b) CD80/PA6; (c) CD68/PA6; (d) CD57/PA6.

analysis (DMTA) is more sensitive to the glass transition than DSC. In DMTA, it is generally accepted that the temperature at the maximum value in $\tan \delta$ corresponds to the glass-rubber transition. Figure 5a shows plots of $\tan \delta$ versus temperature for CD95/PA6 blends. Primary transitions of pure chitosans appear around $140^{\circ} \mathrm{C}$ in $\tan \delta$. This peak may correspond to the $\alpha$ relaxation of chitosan. Ratto ${ }^{27}$ also reported that the glass-rubber transition of chitosan was observed at $140^{\circ} \mathrm{C}$ by DMTA. A shoulder around $0^{\circ} \mathrm{C}$ is attributed to the water induced

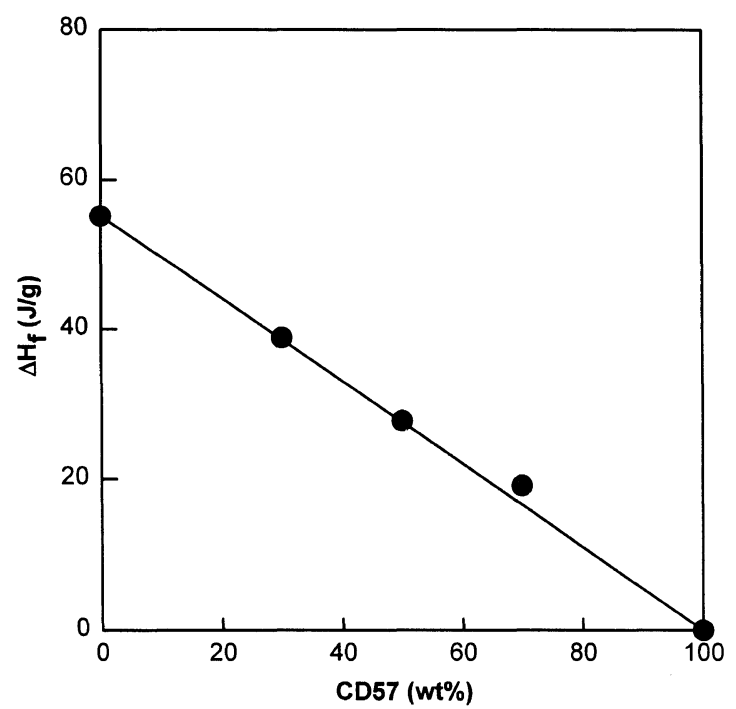

Figure 4. Heat of fusion as a measure of crystallinity in blends of CD57/PA6 determined by differential scanning calorimetry.
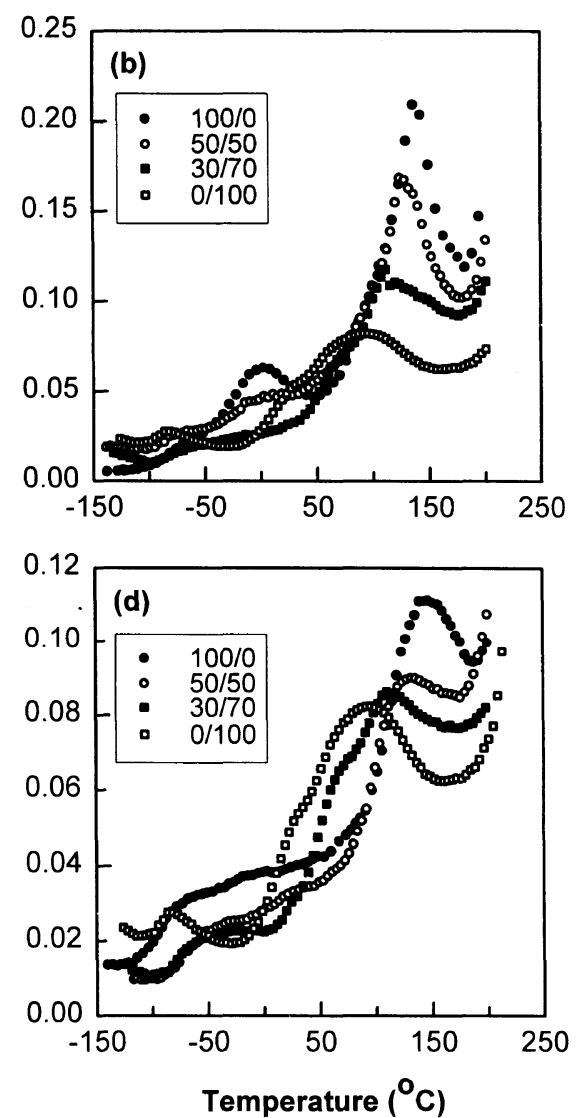

$\beta$ relaxation. The primary dispersion peak of PA6 occurs at $95^{\circ} \mathrm{C}$ in $\tan \delta$. This dispersion is considered to be chain motion in a phase of low regularity such as nematic structure, which behaves in almost the same way as amorphous regions. ${ }^{4,24}$ The shoulder around $40^{\circ} \mathrm{C}$ results from molecular relaxation in completely amorphous regions, and a smaller peak around $-70^{\circ} \mathrm{C}$ is assigned as the $\beta$ relaxation of PA6. ${ }^{4}$ The $\alpha$ dispersion temperature of chitosan in CD95/PA6 blends does not change significantly with the addition of PA6. In CD80/PA6, 


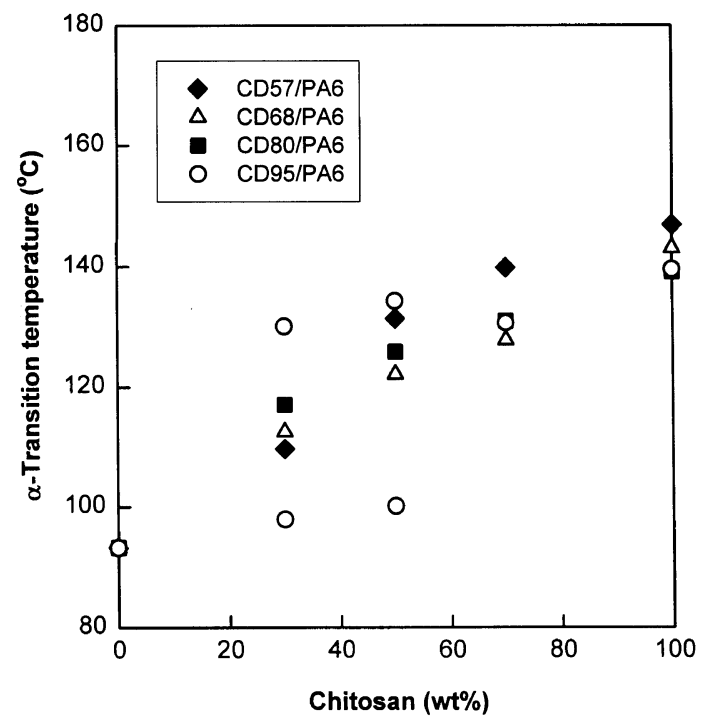

Figure 6. $\alpha$-Transition temperature of chitosan/PA6 blends as a function of composition.

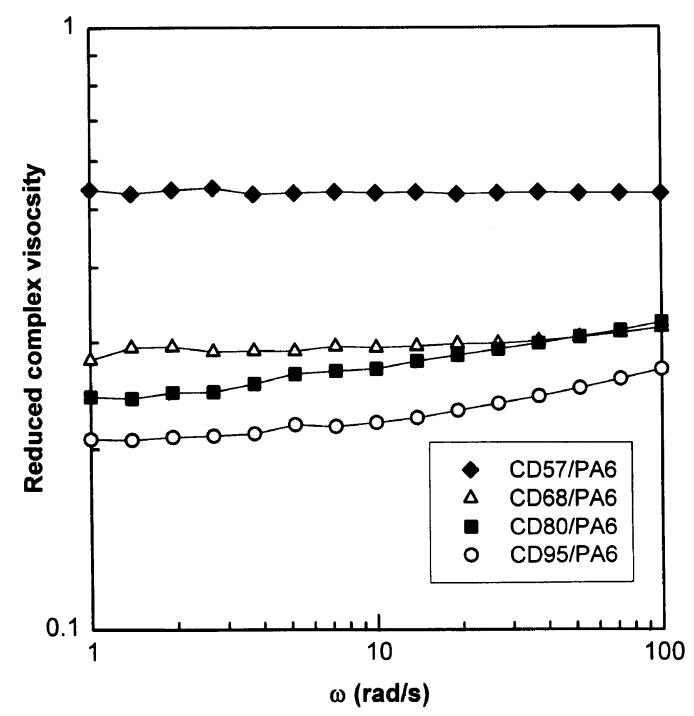

Figure 7. Reduced complex viscosity of $3 \mathrm{wt} \%$ solution in formic acid. Blend ratio was fixed at 70/30 for all chitosan/PA6 blends.

however, the $\alpha$ dispersion peak of chitosan in CD80/PA6 shifts to a lower temperature with increasing PA6 in the blends, as shown in Figure 5b. The shift of $\alpha$ relaxation of chitosan becomes more obvious in CD68/PA6 and CD57/PA6 blends (Figures 5c and 5d). When the $\alpha$ relaxation temperature is plotted against blend composition (Figure 6), the transition temperature of CD68/PA6 and CD57/PA6 blends shows composition-dependence, whereas the transition temperature of CD95/PA6 does not show composition-dependence. These results lead to conclusions that some amorphous portion in PA6 is incorporated into the chitosan phase and that the incorporation becomes more significant as the deacetylation of chitosan decreases.

To find evidence for interaction between chitosans and PA6, complex viscosity $\left(\eta^{*}\right)$ of blend solutions was measured at room temperature in frequency range of $1-100 \mathrm{rads}^{-1}$. The $3 \mathrm{wt} \%$ solution samples were prepared with formic acid. Four chitosans with different $D d$ have different molecular weights. Thus the complex vis-

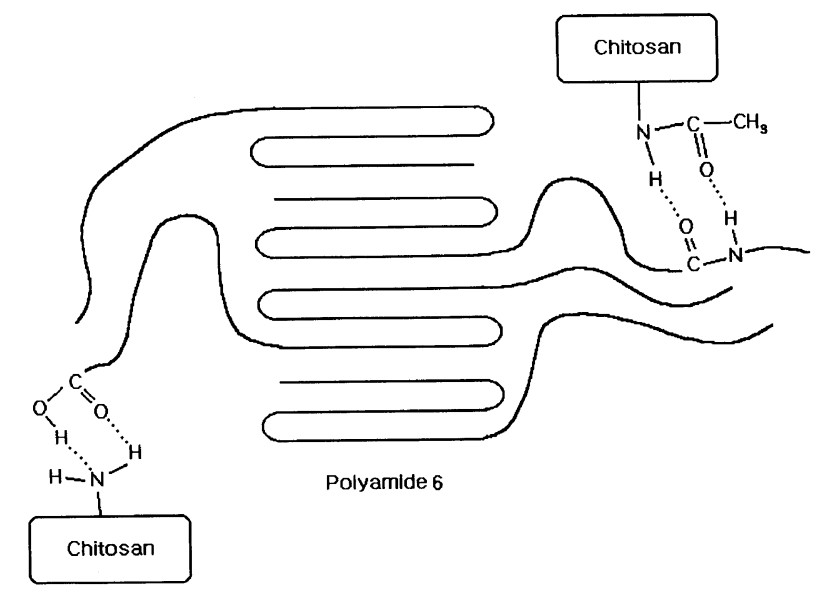

Figure 8. Schematic presentation of hydrogen bonding between chitosan and PA6 in amorphous region.

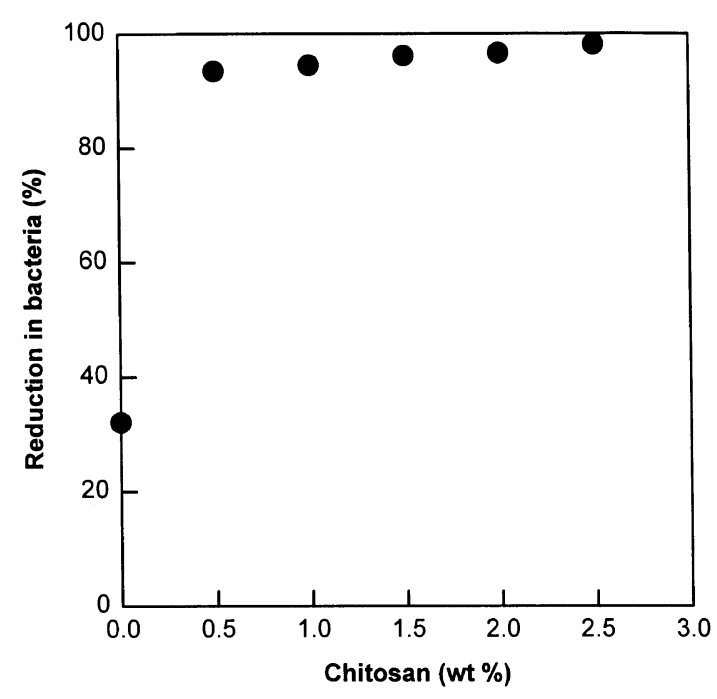

Figure 9. Antimicrobial activity of CD57/PA6.

cosity of blending solutions were normalized with respect to that of corresponding pure chitosan solution to exclude the effects of molecular weight and chain rigidity of chitosan on complex viscosity. As shown in Figure 7, the CD57/PA6 shows the highest value of reduced complex viscosity, and viscosity decreases with increase in the $D d$ of chitosan. Generally the solution viscosity of polymer blends becomes higher, if there are interactions between two components. This suggests that interaction between PA6 and chitosan with lower $D d$ is stronger than that between PA6 and the chitosan with higher $D d$. This is consistent with the results of dynamic mechanical analysis.

From the above results of DMTA and solution viscosity, it is concluded that the miscibility of chitosan/ PA6 blends increases with decreasing $D d$. This may be explained as followings (Figure 8). The chitosan with lower $D d$ retains relatively a large number of acetamide groups in the chitosan molecules, which are able to easily interact with amide groups in the PA6 chains via amideamide hydrogen bonding. The chitosan with higher $D d$ has a relatively small number of amide groups, but it has a large number of amine groups that can interact only with carboxyl end groups in the PA6 chain. There- 
fore, it is more probable for chitosan to interact with PA6 in the former case, resulting in improvement of miscibility between chitosan and PA6. Infrared spectroscopy may sometimes provide direct evidence of polymerpolymer interaction. However, in our system a definite change in spectra was not identified due to the overlap of absorption bands from amide group in PA6 and acetamide group in chitosans.

Since chitosan has a cationic nature while most living tissues have a negative charge, chitosan may be able to bind to most tissues, skin, bones and hair. Thus binding of chitosan to living cell becomes very important in biomedical applications. ${ }^{28}$ In Figure 9, which shows the antimicrobial activity of CD57/PA6 blend, the growth of bacteria is almost completely suppressed in the presence of $0.5 \mathrm{wt} \%$ chitosan in blends. It is expected that the antimicrobial activity will be enhanced due to the higher density of amine groups, if chitosan with a high $D d$ is used in blend samples. Thus, it may be concluded that all blend films used in this study show excellent antimicrobial activity.

\section{CONCLUSIONS}

The miscibility of chitosan/PA6 blends was examined as a function of the $D d$ of chitosan. From X-ray and DSC analysis, no significant change is observed in PA6 crystalline regions regardless of the $D d$ in chitosan, suggesting that chitosan does not hinder the crystallization of PA6 in the blends. On the other hand, the $\alpha$ relaxation of chitosan from DMTA shifted to lower temperature with the addition of PA6, and the shift becomes larger as the $D d$ in chitosan decreases. The complex viscosity of blending solution increases with decreasing deacetylation. Interactions would thus appear to occur between amorphous regions of both polymers and the blend miscibility increases with decreasing deacetylation in chitosan.

\section{REFERENCES}

1. Y. Nishio and R. St. J. Manley, Macromolecules, 21, 1270 (1988).

2. Y. Nishio and R. St. J. Manley, Macromolecules, 22, 2547 (1989).

3. Y. Nishio, S. K. Roy, and R. St. J. Manley, Polymer, 28, 1385 (1987).

4. Y. Nishio and R. St. J. Manley, Polym. Eng. Sci., 30, 71 (1990).

5. B. Schartel, J. Wendling, and J. H. Wendorff, Macromolecules, 29, 1521 (1996).

6. D. Radloff, C. Boeffel, and H. W. Spiess, Macromolecules, 29, 1528 (1996).

7. J. A. Ratto, C. C. Chen, and R. B. Blumstein, J. Appl. Polym. Sci., 59, 1451 (1996).

8. M. Garcia-ramirez, J. Y. Caraille, D. Dupeyre, and A. Peguy, J. Polym. Sci., Part B, Polym. Phys., 32, 1437 (1994).

9. M. Garcia-ramirez, J. Y. Caraille, A. Dufresne, and P. Tekely, J. Polym. Sci., Part B, Polym. Phys., 33, 2109 (1995).

10. M. Garcia-ramirez, J. Y. Caraille, A. Dufresne, and D. Dupeyre, J. Appl. Polym. Sci., 59, 1995 (1996).

11. R. A. A. Muzzarelli, "Chitin," Pergamon Press, Oxford, 1977.

12. R. A. A. Muzzarelli, "Natural Chelating Polymers," Pergamon Press, Oxford, 1973.

13. H. S. Blair, J. Guthrie, T. Law, and P. Turkington, J. Appl. Polym. Sci., 33, 641 (1987).

14. M. T. Qurashi, H. S. Blair, and S. J. Allen, J. Appl. Polym. Sci., 46, 255 (1992)

15. W. Zhao, L. Yu, X. Zhong, Y. Zhang, and J. Sun, J. Macromol. Sci. Phys., B34(3), 231 (1955).

16. S. Aibi, Int. J. Biol. Macromol., 14, 225 (1992).

17. G. G. Maghami and G. A. F. Roberts, Makromol. Chem., 189, 195 (1988).

18. S. Mima, M. Miya, R. Iwamoto, and S. Yoshikawa, J. Appl. Polym. Sci., 28, 1809 (1981).

19. J. G. Domszy and G. A. F. Roberts, Makromol. Chem., 186, 1671 (1985).

20. K. Sakurai, M. Takai, and T. Takahashi, Sen-i Gakkaishi, 40 , T-246 (1984).

21. "Dow Corning Test Method," CTM 0923, 1979.

22. R. J. Samuels, J. Polvm. Sci., Polym. Phys. Ed., 19, 1081 (1981).

23. D. R. Holmes, C. Bunn, and D. J. Smith, J. Polym. Sci., 17, 159 (1955).

24. Y. Nishio, H. Suzuki, and K. Morisaki, Polym. International, 31 , 15 (1993).

25. M. Todoki and T. Kawaguchi, J. Polym. Sci., Polym. Phys. Ed., 15, 1067 (1977).

26. M. Kyotani, J. Polym. Sci., Polym. Phys. Ed., 17, 103 (1979).

27. J. Ratto, Ph. D. Thesis, University of Massachusetts, Lowell, 1993.

28. H. L. Nossel, G. D. Wilner, and E. C. Leoy, Nature, 221, 759 (1969). 